\title{
A Potassium Tungsten Oxyfluoride with Strong Second-Harmonic Generation Response Derived From Anion-Ordered Functional Motif
}

\author{
Hong-Xin Tang ${ }^{1,2,4}$, Rui-Biao $\mathrm{Fu}^{1,2^{*}}, \mathrm{Zu}-\mathrm{Ju} \mathrm{Ma}^{3 *}$, and Xin-Tao $\mathrm{Wu}^{1}$ \\ 1 State Key Laboratory of Structural Chemistry, Fujian Institute of Research on the Structure of Matter, \\ Chinese Academy of Sciences, Fuzhou, Fujian 350002, P. R. China. \\ 2 Fujian Science \&Technology InnovationLaboratory for Optoelectronic Information of China, Fuzhou, \\ Fujian 350108, P. R. China. \\ 3 School of Environmental and Materials Engineering, Yantai University, Yantai, Shandong 264005, P. \\ R. China. \\ 4 University of Chinese Academy of Sciences, Beijing, 100049, P. R. China.
}

Corresponding author: *Rui-Biao Fu, furb@fjirsm.ac.cn;*Zu-Ju Ma, zjma@outlook.com. 


\section{DFT calculations}

In the static case, the imaginary part of the static second-order optical susceptibility can be expressed as:

$$
\begin{aligned}
& \chi^{a b c} \\
& =\frac{e^{3}}{\mathrm{~h}^{2} \Omega} \sum_{n m l, k} \frac{r_{n m}^{a}\left(r_{m l}^{b} r_{l n}^{c}+r_{m l}^{c} r_{l n}^{b}\right)}{2 \omega_{n m} \omega_{m l} \omega_{l n}}\left[\omega_{n} f_{m l}+\omega_{m} f_{l n}+\omega_{l} f_{n m}\right] \\
& +\frac{i e^{3}}{4 \mathrm{~h}^{2} \Omega} \sum_{n m, k} \frac{f_{n m}}{\omega_{m n}^{2}}\left[r_{n m}^{a}\left(r_{m n ; c}^{b}+r_{m n ; b}^{c}\right)+r_{n m}^{b}\left(r_{m n ; c}^{a}+r_{m n ; a}^{c}\right)+r_{n m}^{c}\left(r_{m n ; b}^{a}+r_{m n ; a}^{b}\right)\right]
\end{aligned}
$$

where $r$ is the position operator, $\hbar \omega_{n m}=\hbar \omega_{n}-\hbar \omega_{m}$ is the energy difference for the bands $m$ and $n$, $f_{m n}=f_{m}-f_{n}$ is the difference of the Fermi distribution functions, subscripts $a, b$, and $c$ are Cartesian indices, and $r_{m n ; a}^{b}$ is the so-called generalized derivative of the coordinate operator in $k$ space,

$r_{n m ; a}^{b}=\frac{r_{n m}^{a} \Delta_{m n}^{b}+r_{n m}^{b} \Delta_{m n}^{a}}{\omega_{n m}}+\frac{i}{\omega_{n m}} \times \sum_{l}\left(\omega_{l m} r_{n l}^{a} r_{l m}^{b}-\omega_{n l} r_{n l}^{b} r_{l m}^{a}\right)$

where $\Delta^{a}{ }_{n m}=\left(p_{n n}^{a}-p^{a}{ }_{m m}\right) / m$ is the difference between the electronic velocities at the bands $n$ and $m$. The $\chi^{(2)}$ coefficients here also were calculated from PBE wave functions with a $4 \times 8 \times 2$ k-point grid and about 200 bands. A scissor operator has been added to correct the conduction band energy (corrected to the experimental gap), which has been proved to be reliable in predicting the second order susceptibility for semiconductors and insulators. ${ }^{1,2}$ 
Table S1. NLO properties of selected NCS alkali/alkaline earth metals $\mathrm{d}^{0}$ transition metal oxyfluorides.

\begin{tabular}{|c|c|c|c|c|c|c|c|}
\hline Compounds & $\begin{array}{l}\text { Space } \\
\text { group }\end{array}$ & $\begin{array}{c}\text { Band gap } \\
(\mathrm{eV})\end{array}$ & $\begin{array}{c}\text { SHG } \\
(1064 \mathrm{~nm})\end{array}$ & $\left\langle\mathrm{d}_{\mathrm{ij}}\right\rangle_{\text {theo }}(\mathrm{pm} / \mathrm{V})$ & $\begin{array}{c}\text { Birefringence } \\
(1064 \mathrm{~nm})\end{array}$ & $\begin{array}{c}\text { PM/NPM[a] } \\
(1064 n m)\end{array}$ & Ref \\
\hline $\mathrm{K}_{3} \mathrm{WO}_{3} \mathrm{~F}_{3}$ & $\mathrm{Cm}$ & 4.315 & $\begin{array}{c}\text { No noticeable } \\
\text { signal }\end{array}$ & $\mathrm{d}_{12}=3.01, \mathrm{~d}_{33}=2.91$ & 0.035 & NPM & 3 \\
\hline $\mathrm{Ba}_{2} \mathrm{MoO}_{3} \mathrm{~F}_{4}$ & $C c$ & 3.45 & $8 \times \alpha-\mathrm{SiO}_{2}$ & $\mathrm{~d}_{33}=-5.7, \mathrm{~d}_{13}=-3.47$ & 0.1052 & - & 4,5 \\
\hline $\mathrm{Ba}_{2} \mathrm{WO}_{3} \mathrm{~F}_{4}$ & $C c$ & 4.13 & $5.1 \times \alpha-\mathrm{SiO}_{2}$ & $\mathrm{~d}_{33}=-5.7, \mathrm{~d}_{11}=2.96$ & 0.0856 & - & 5,6 \\
\hline $\mathrm{NaVOF}_{4} \cdot \mathrm{H}_{2} \mathrm{O}$ & $\operatorname{Pna}_{1}$ & - & - & $\left\langle\mathrm{d}_{\mathrm{eff}}\right\rangle_{\exp }=0.55$ & - & NPM & 7 \\
\hline $\begin{array}{c}\mathrm{NaVO}_{2-\mathrm{x}} \mathrm{F}_{2+\mathrm{x}} \\
(\mathrm{x}=1 / 3)\end{array}$ & $P 2_{1}$ & 0.632 & - & $\left\langle\mathrm{d}_{\mathrm{eff}}\right\rangle_{\exp }=1.15$ & - & $\mathrm{PM}$ & 7 \\
\hline $\mathrm{KNaNbOF}_{5}$ & $P n a 2_{1}$ & 5.65 & $0.6 \times \mathrm{KDP}$ & $\mathrm{d}_{33}=0.72, \mathrm{~d}_{31}=-0.44$ & 0.0447 & $\mathrm{PM}$ & 5,8 \\
\hline $\mathrm{K}_{2} \mathrm{TiOF}_{4}$ & $P n a 2_{1}$ & 3.21 & - & $\mathrm{d}_{32}=0.62, \mathrm{~d}_{31}=0.10$ & 0.1468 & - & 5,9 \\
\hline $\mathrm{Li}_{2} \mathrm{Ta}_{2} \mathrm{O}_{3} \mathrm{~F}_{6}$ & $P 3_{1} 21$ & 5.49 & - & $\mathrm{d}_{11}=0.15$ & 0.0948 & - & 5,10 \\
\hline $\mathrm{SrMoO}_{2} \mathrm{~F}_{4}$ & $P c a 2_{1}$ & 3.42 & $1.1 \times \alpha-\mathrm{SiO}_{2}$ & - & - & $\mathrm{PM}$ & 11 \\
\hline$\alpha-\mathrm{BaMoO}_{2} \mathrm{~F}_{4}$ & $P c a 2_{1}$ & 3.30 & $0.7 \times \alpha-\mathrm{SiO}_{2}$ & - & - & $\mathrm{PM}$ & 11 \\
\hline $\mathrm{PbMoO}_{2} \mathrm{~F}_{4}$ & $P c a 2_{1}$ & 3.20 & $0.5 \times \alpha-\mathrm{SiO}_{2}$ & - & - & $\mathrm{PM}$ & 11 \\
\hline $\mathrm{KWO}_{3} \mathrm{~F}$ & $A m a 2$ & 2.68 & $3 \times \mathrm{KDP}$ & $\mathrm{d}_{15}=2.72, \mathrm{~d}_{32}=1.51$ & 0.088 & $\mathrm{PM}$ & $\begin{array}{l}\text { This } \\
\text { work }\end{array}$ \\
\hline
\end{tabular}

[a] PM: Phase-Matching, NPM: No Phase-Matching; “ -" means no data available.

Table S2. Atomic coordinates, equivalent isotropic displacement parameters $\left(\AA^{2}\right)$ and BVS of $\mathrm{KWO}_{3} \mathrm{~F} . \mathrm{U}_{\mathrm{eq}}$ is defined as one-third of the trace of the orthogonalized $\mathrm{U}_{\mathrm{ij}}$ tensor.

\begin{tabular}{cccccccc}
\hline Atom & $\begin{array}{c}\text { Wyckof } \\
\mathrm{f}\end{array}$ & $\mathrm{x}$ & $\mathrm{y}$ & $\mathrm{z}$ & $\mathrm{U}_{\text {eq }}$ & $\mathrm{BVS}$ & Occ. \\
\hline $\mathrm{W} 1$ & $4 \mathrm{~b}$ & 0.75000 & $0.04766(17)$ & $-0.00341(2)$ & $0.00950(3)$ & 6.35 & 1.0 \\
$\mathrm{~K} 1$ & $4 \mathrm{a}$ & 1.00000 & 0 & $-0.29250(7)$ & $0.01920(13)$ & 0.89 & 1.0 \\
$\mathrm{O} 1$ & $4 \mathrm{~b}$ & & $0.02400(110$ & $-0.13600(30)$ & $0.02000(8)$ & 2.01 & 1.0 \\
& & 0.75000 & ) & & & \\
$\mathrm{O} 2$ & $4 \mathrm{~b}$ & 0.75000 & $0.51700(70)$ & $0.01300(30)$ & $0.05300(13)$ & 2.12 & 1.0 \\
$\mathrm{O} 3$ & $4 \mathrm{a}$ & 1.00000 & 0 & $0.01800(30)$ & $0.04600(9)$ & 2.15 & 1.0 \\
$\mathrm{~F} 1$ & $4 \mathrm{~b}$ & 0.75000 & $0.00700(80)$ & $0.16000(20)$ & $0.02300(6)$ & 0.96 & 1.0 \\
\hline
\end{tabular}

Table S3. Selected bond lengths $(\AA)$ of $\mathrm{KWO}_{3} \mathrm{~F}$.

\begin{tabular}{llll}
\hline Bond & Bond lengths & Bond & Bond lengths \\
\hline $\mathrm{W}(1)-\mathrm{O}(1)$ & $1.735(3)$ & $\mathrm{K}(1)-\mathrm{O}(1)$ & $2.769(3)$ \\
$\mathrm{W}(1)-\mathrm{O}(2)$ & $1.802(3)$ & $\mathrm{K}(1)-\mathrm{O}(1) \# 2$ & $2.769(3)$ \\
$\mathrm{W}(1)-\mathrm{O}(2) \# 1$ & $2.034(3)$ & $\mathrm{K}(1)-\mathrm{F}(1) \# 3$ & $2.718(2)$ \\
$\mathrm{W}(1)-\mathrm{O}(3)$ & $1.893(5)$ & $\mathrm{K}(1)-\mathrm{F}(1) \# 4$ & $2.756(2)$ \\
$\mathrm{W}(1)-\mathrm{O}(3) \# 2$ & $1.893(5)$ & $\mathrm{K}(1)-\mathrm{F}(1) \# 5$ & $2.718(2)$ \\
$\mathrm{W}(1)-\mathrm{F}(1)$ & $2.141(3)$ & $\mathrm{K}(1)-\mathrm{F}(1) \# 6$ & $2.756(2)$ \\
\hline
\end{tabular}


Symmetry codes: \#1 x, -1+y, z; \#2 3/2-x, y, z; \#3 x, -1/2+y, -1/2+z; \#4 x, 1/2+y, -1/2+z \#5 2-x, 1/2-y, -1/2+z; \#6 2-x, -1/2-y, -1/2+z.

Table S4. Selected bond angles (deg.) of $\mathrm{KWO}_{3} \mathrm{~F}$.

\begin{tabular}{llll}
\hline Bond & Bond angles & Bond & Bond angles \\
\hline $\mathrm{O}(1)-\mathrm{W}(1)-\mathrm{F}(1)$ & $172.8(9)$ & $\mathrm{O}(3)-\mathrm{W}(1)-\mathrm{O}(3) \# 2$ & $160.0(16)$ \\
$\mathrm{O}(2)-\mathrm{W}(1)-\mathrm{O}(2) \# 1$ & $167.0(2)$ & & \\
\multicolumn{2}{l}{ Symmetry codes: $\# 1 \mathrm{x},-1+\mathrm{y}, \mathrm{z} ; \# 2$ 3/2-x, y, z. } & & \\
\hline
\end{tabular}

Table S5. Selected bond lengths, experimental bond valences, theoretical bond valences and BSI and GII indices for $\mathrm{KWO}_{3} \mathrm{~F}$.

\begin{tabular}{|c|c|c|c|c|c|c|c|c|}
\hline & & \multicolumn{2}{|l|}{ W1 } & \multicolumn{3}{|c|}{$\mathrm{K} 1$} & $\begin{array}{l}V_{i}= \\
z_{i}=\end{array}$ & $\begin{array}{l}\sum_{i} S_{i j} \\
\sum_{i} s_{i j}\end{array}$ \\
\hline \multirow[t]{3}{*}{$\mathrm{O} 1$} & $R_{i j}$ & 1.74 & & 2.77 & 2.77 & & & \\
\hline & $S_{i j}$ & 1.65 & & 0.18 & 0.18 & & 2.01 & \\
\hline & $s_{i j}$ & 1.18 & & 0.41 & 0.41 & & 2.00 & \\
\hline \multirow[t]{3}{*}{$\mathrm{O} 2$} & $R_{i j}$ & 1.80 & 2.03 & & & & & \\
\hline & $S_{i j}$ & 1.38 & 0.74 & & & & 2.12 & \\
\hline & $s_{i j}$ & 1.00 & 1.00 & & & & 2.00 & \\
\hline \multirow[t]{3}{*}{$\mathrm{O} 3$} & $R_{i j}$ & 1.89 & 1.89 & & & & & \\
\hline & $S_{i j}$ & 1.08 & 1.08 & & & & 2.15 & \\
\hline & $s_{i j}$ & 1.00 & 1.00 & & & & 2.00 & \\
\hline \multirow[t]{3}{*}{ F1 } & $R_{i j}$ & 2.14 & & 2.72 & 2.76 & 2.76 & & \\
\hline & $S_{i j}$ & 0.43 & & 0.14 & 0.13 & 0.13 & 0.96 & \\
\hline & $s_{i j}$ & 0.82 & & 0.05 & 0.05 & 0.05 & 1.00 & \\
\hline$V_{j}=$ & $\sum S_{i j}$ & 6.35 & & & 0.8 & & $\mathrm{BSI}=$ & 0.25 \\
\hline$z_{j}=$ & $\sum_{j} s_{i j}$ & 6.00 & & & 1.0 & & $\mathrm{GII}=$ & 0.17 \\
\hline \multicolumn{9}{|c|}{$\begin{array}{l}* R_{i j} \text {, selected bond length } i j ; S_{i j} \text {, experimental bond valence (vu); } s_{i j} \text {, theoretical bond valence (vu), calculated by solving the } \\
\text { network equations dependent on the methods described by Brown; } ;{ }^{12}, V_{i}, V_{j} \text {, experimental valences of anions } i \text { and cations } j ; z_{i} \\
z_{j} \text {, calculated theoretical valence of anions } i \text { and cations } j ; \text { BSI }=\left[\left\langle\left(S_{i}-s_{i j}\right)^{2}\right]^{12} \text {, bond strain index; GII }=\left[\left\langle\left(V_{i, j}-z_{i, j}\right)^{2}\right\rangle\right]^{1 / 2} \text {, globa }\right. \\
\text { instability index; }\langle\rangle \text { means an average value of all bonds in this formula unit. A BSI or GII value greater than } 0.05 \text { indicates } \\
\text { structure is strained. And a GII value greater than } 0.20 \text { indicates it is unstable. All showed data were kept two decimals anc } \\
\text { rounding off. }\end{array}$} \\
\hline \multicolumn{9}{|c|}{$\begin{array}{l}\text { Table S6. Calculation of dipole moment of octahedron, net dipole moment in one unit cell and the } \\
\text { dipole moment per unit volume in } \mathrm{KWO}_{3} \mathrm{~F} \text {. }\end{array}$} \\
\hline \multirow{2}{*}{\multicolumn{2}{|c|}{ Compound }} & \multirow[t]{2}{*}{ Octahedron } & \multicolumn{3}{|c|}{ Dipole moment (D) } & \multirow{2}{*}{$\begin{array}{c}\text { Net dipole } \\
\text { moment (D)(one } \\
\text { unit cell) }\end{array}$} & \multirow{2}{*}{\multicolumn{2}{|c|}{$\begin{array}{l}\text { Dipole moment } \\
\text { per unit volume } \\
\qquad\left(\mathrm{D} \AA^{-3}\right)\end{array}$}} \\
\hline & & & $a$-axis & $b$-axis & $c$-axis & & & \\
\hline \multirow{4}{*}{\multicolumn{2}{|c|}{$\mathrm{KWO}_{3} \mathrm{~F}$}} & $\mathrm{~W} \# 1 \mathrm{O}_{5} \mathrm{~F}$ & 0 & -0.94 & -0.96 & \multirow{4}{*}{-3.84} & \multirow{4}{*}{\multicolumn{2}{|c|}{-0.01}} \\
\hline & & $\mathrm{WO}_{5} \mathrm{~F}$ & 0 & 0.94 & -0.96 & & & \\
\hline & & $\mathrm{W} \# 2 \mathrm{O}_{5} \mathrm{~F}$ & 0 & -0.94 & -0.96 & & & \\
\hline & & $\mathrm{W} \# 3 \mathrm{O}_{5} \mathrm{~F}$ & 0 & 0.94 & -0.96 & & & \\
\hline
\end{tabular}

Symmetry codes: \#1 1-x, 1-y, z; \#2 1-x, 0.5-y, 0.5+z; \#3 x, 0.5+y, 0.5+z. 


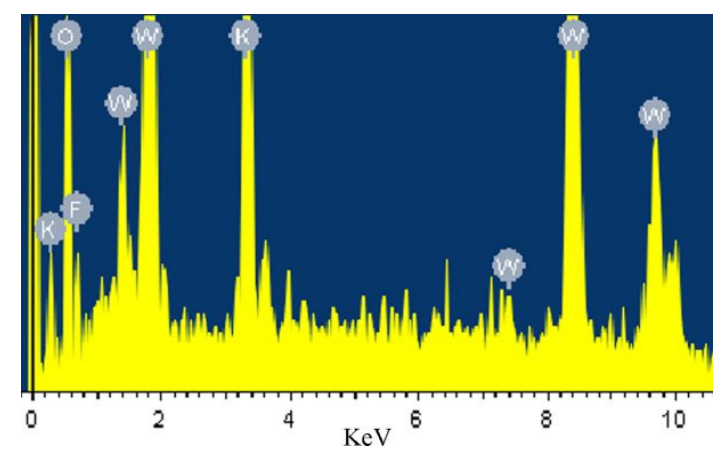

Figure S1. EDS spectrum of $\mathrm{KWO}_{3} \mathrm{~F}$.

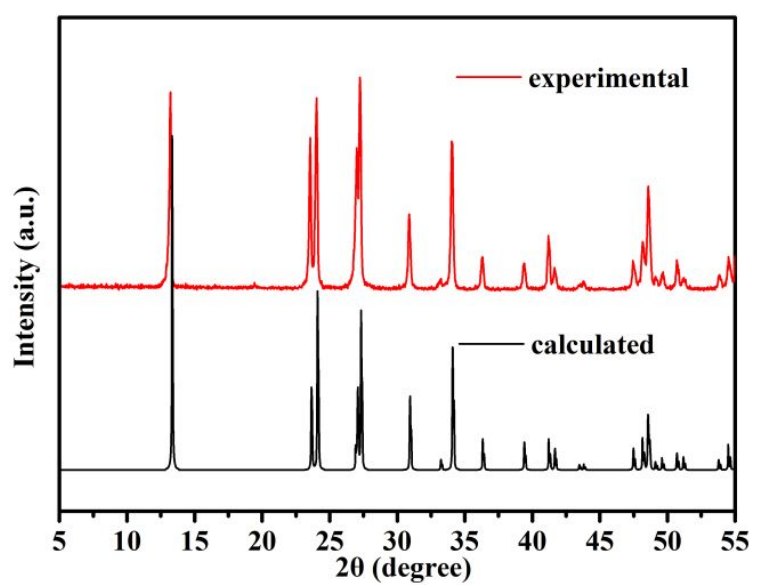

Figure S2. PXRD pattern of $\mathrm{KWO}_{3} \mathrm{~F}$. 


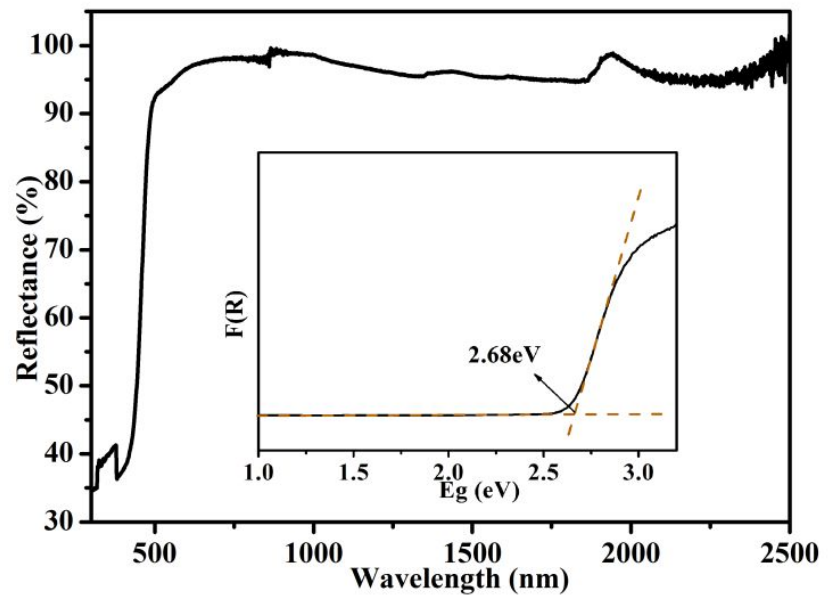

Figure S3. UV-vis-NIR diffuse reflectance spectrum of $\mathrm{KWO}_{3} \mathrm{~F}$.

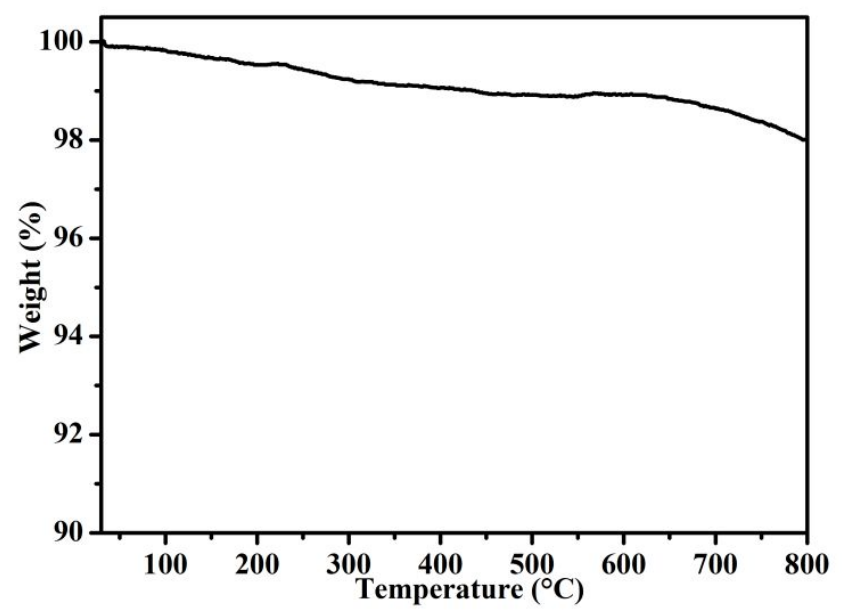

Figure S4. TG curve of $\mathrm{KWO}_{3} \mathrm{~F}$. 


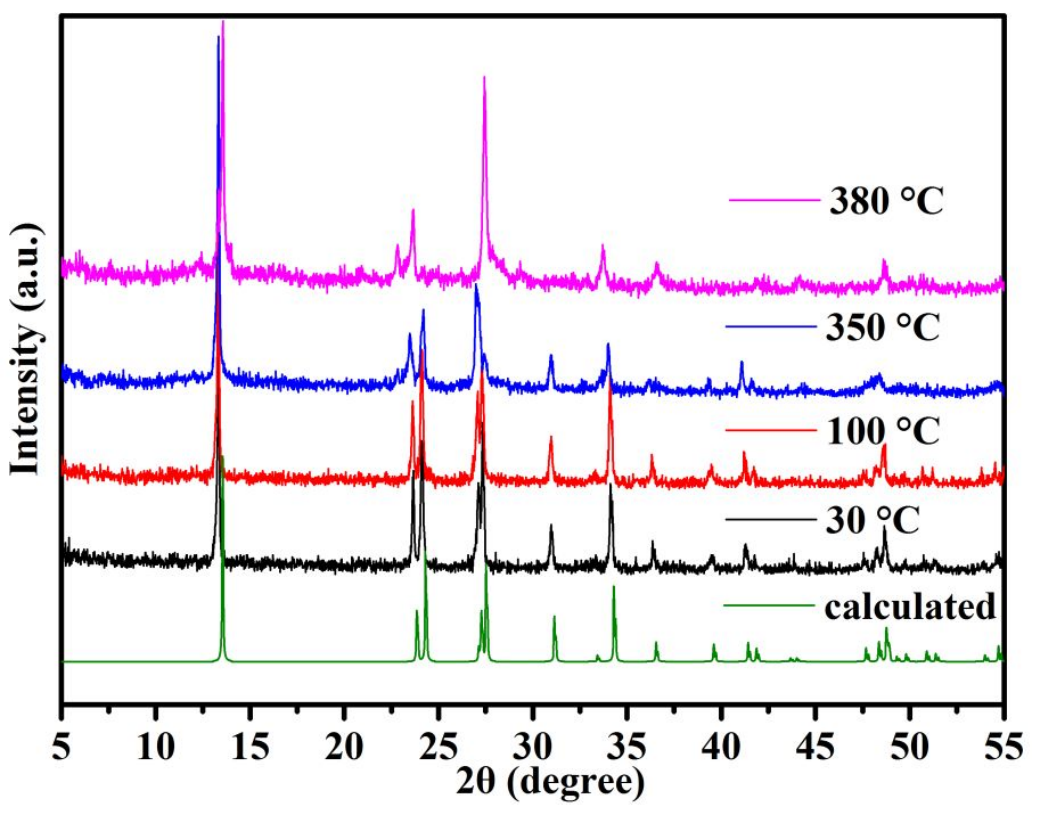

Figure S5. Variable temperature PXRD patterns of $\mathrm{KWO}_{3} \mathrm{~F}$.

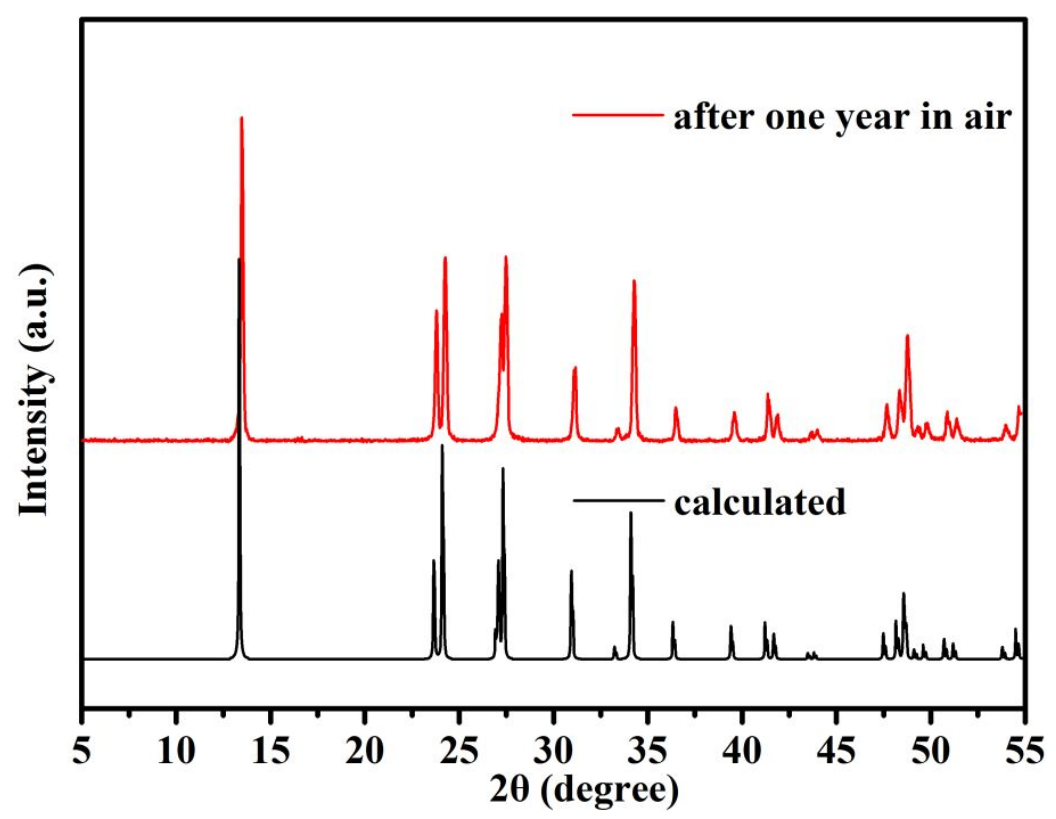

Figure S6. PXRD patterns of $\mathrm{KWO}_{3} \mathrm{~F}$ after one year in air. 


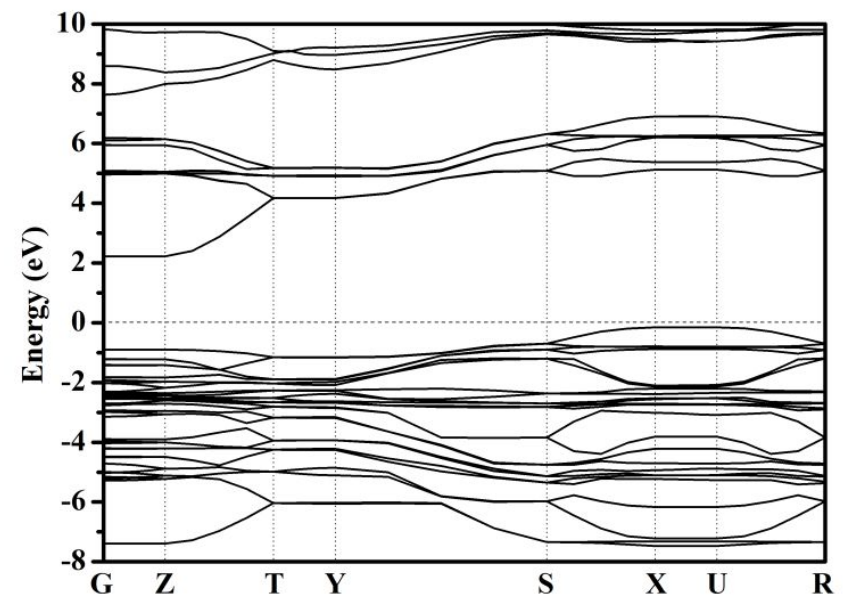

Figure S7. The calculated band structure of $\mathrm{KWO}_{3} \mathrm{~F}$.

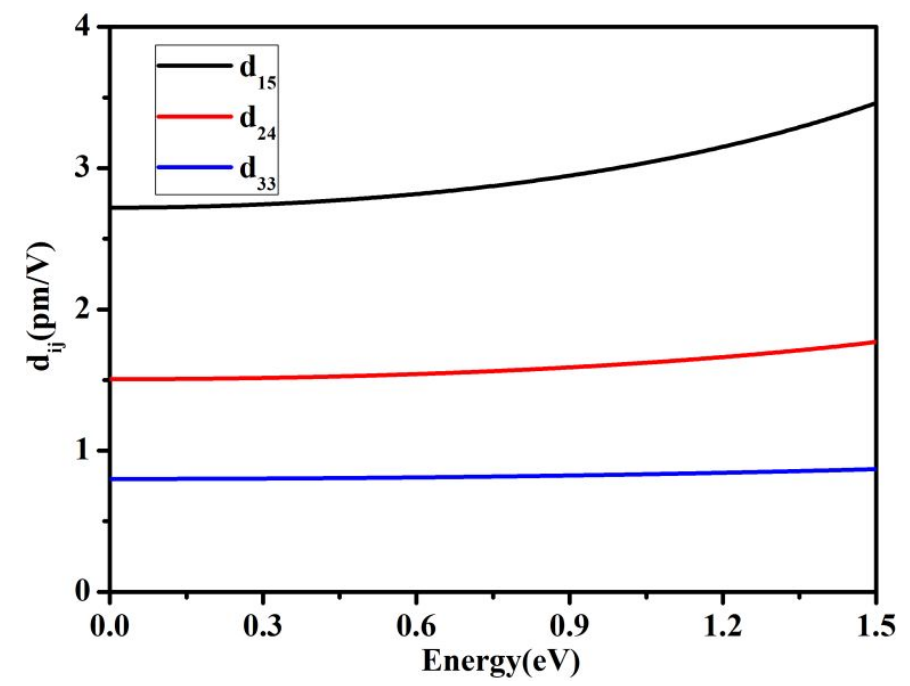

Figure S8. The frequency-dependent second harmonic generation (SHG) coefficients of $\mathrm{KWO}_{3} \mathrm{~F}$. 


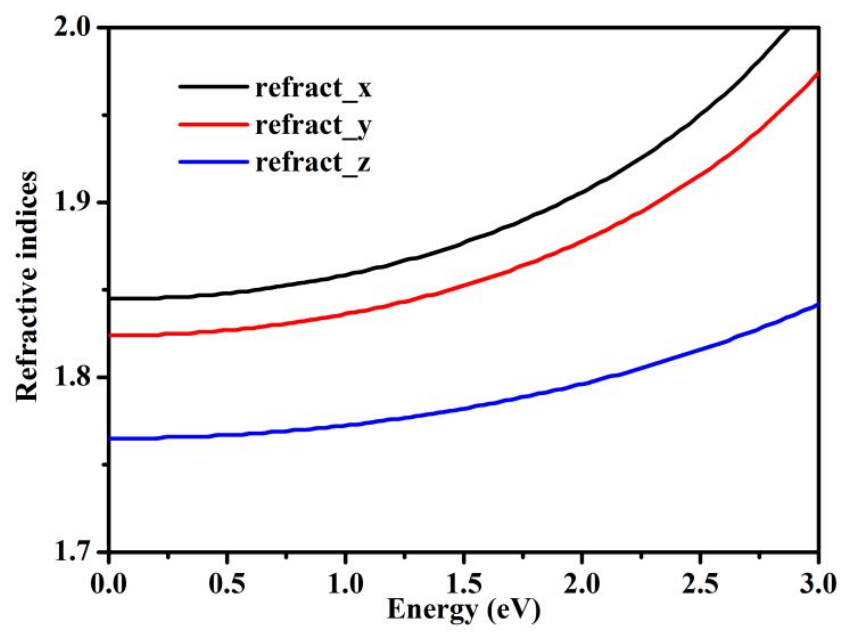

Figure S9. The calculated frequency-dependent refractive indices of $\mathrm{KWO}_{3} \mathrm{~F}$.

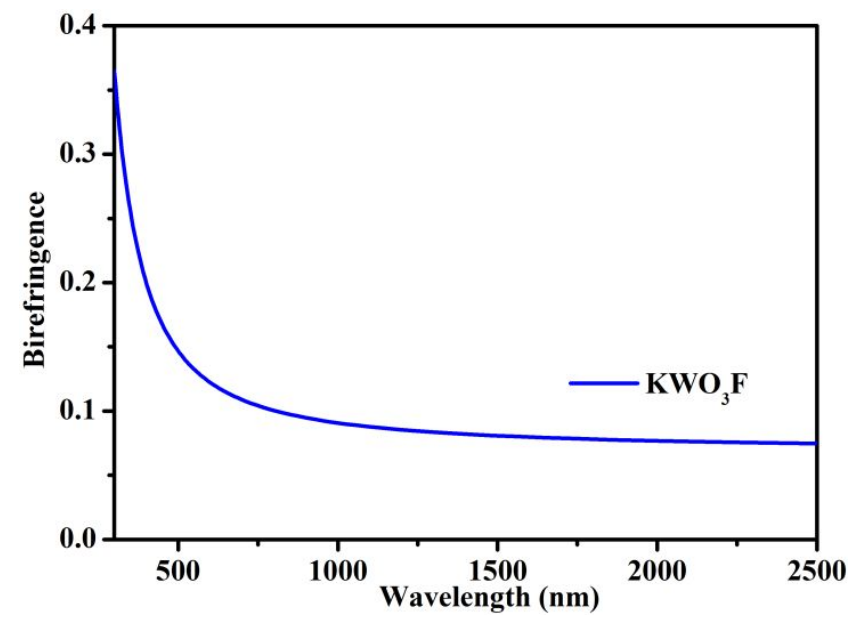

Figure S10. Birefringence of $\mathrm{KWO}_{3} \mathrm{~F}$.

\section{References}

(1) Champagne, B.; Bishop, D. M. Calculations of Nonlinear Optical Properties for the Solid State. Adv. Chem. Phys. 2003, 126, 41-92.

(2) Reshak, A. H.; Auluck, S.; Kityk, I. V. Specific Features in the Band Structure and Linear and Nonlinear Optical Susceptibilities of $\mathrm{La}_{2} \mathrm{CaB}_{10} \mathrm{O}_{19}$ Crystals. Physical Review B 2007, 75, 245120.

(3) Atuchin, V.; Isaenko, L.; Kesler, V.; Lin, Z.; Molokeev, M.; Yelisseyev, A.; Zhurkov, S. Exploration on Anion Ordering, Optical Properties and Electronic Structure in $\mathrm{K}_{3} \mathrm{WO}_{3} \mathrm{~F}_{3}$ Elpasolite. $J$. Solid State Chem. 2012, 187, 159-164.

(4) Wiegel, M.; Blasse, G. Luminescence and Nonlinear Optical Properties of Bariumfluoromolybdate $\left(\mathrm{Ba}_{2} \mathrm{MoO}_{3} \mathrm{~F}_{4}\right)$. Solid State Commun. 1993, 86, 239-241.

(5) Huang, J.; Guo, S.; Zhang, Z.; Yang, Z.; Pan, S. Designing Excellent Mid-Infrared Nonlinear Optical Materials with Fluorooxo-Functional Group of $\mathrm{d}^{0}$ Transition Metal Oxyfluorides. Sci. China Mater. 2019, 62, 1798-1806.

(6) Torardi, C.; Brixner, L. Structure and Luminescence of $\mathrm{Ba}_{2} \mathrm{WO}_{3} \mathrm{~F}_{4}$. Mater. Res. Bull. 1985, 20, 137-145.

(7) Donakowski, M. D.; Gautier, R.; Lu, H.; Tran, T. T.; Cantwell, J. R.; Halasyamani, P. S.; 
Poeppelmeier, K. R. Syntheses of Two Vanadium Oxide-Fluoride Materials That Differ in Phase Matchability. Inorg. Chem. 2015, 54, 765-772.

(8) Marvel, M. R.; Lesage, J.; Baek, J.; Halasyamani, P. S.; Stern, C. L.; Poeppelmeier, K. R. CationAnion Interactions and Polar Structures in the Solid State. J. Am. Chem. Soc. 2007, 129, 13963-13969.

(9) Sheng, J.; Tang, K.; Cheng, W.; Wang, J.; Nie, Y.; Yang, Q. Controllable Solvothermal Synthesis and Photocatalytic Properties of Complex (Oxy) Fluorides $\mathrm{K}_{2} \mathrm{TiOF}_{4}, \mathrm{~K}_{3} \mathrm{TiOF}_{5}, \mathrm{~K}_{7} \mathrm{Ti}_{4} \mathrm{O}_{4} \mathrm{~F}_{7}$ and $\mathrm{K}_{2} \mathrm{TiF}_{6}$. J. Hazard. Mater. 2009, 171, 279-287.

(10) Kaskel, S.; Strähle, J. Synthese Und Struktur Von $\mathrm{Li}_{2} \mathrm{Ta}_{2} \mathrm{O}_{3} \mathrm{~F}_{6}$. Z. Anorg. Allg. Chem. 1997, 623, 456-460.

(11) Jo, H.; Lee, M. H.; Ok, K. M. Order and Disorder: Toward the Thermodynamically Stable $\alpha-\mathrm{BaMoO}_{2} \mathrm{~F}_{4}$ from the Metastable Polymorph. Chem. Mater. 2021, 33, 1875-1882.

(12) Brown, I. D. Recent Developments in the Methods and Applications of the Bond Valence Model. Chem. Rev. 2009, 109, 6858-6919.

(13) Brown, I. D. The Chemical Bond in Inorganic Chemistry: The Bond Valence Model. 2006. 